\title{
Integrated OBD-II and mobile application for electric vehicle (EV) monitoring system
}

\begin{abstract}
Electric Vehicles (EV) are becoming more popular, advanced and connected to the internet, the users are being encouraged to use new technologies for tracking, monitoring and controlling their EVs. The experienced users tend to monitor and control specific EV parts including ABS, Airbag, and Air Condition easily and seamlessly. Currently, there is no a distinct system at all that would integrate all these factors into one disposable application. This research develops a system which communicates with On Board Diagnostics of Electric Vehicle by using Controller Area Network communication protocol. The paper discusses how to collect CAN data by new and low-cost method through OBD-II port. The OBD-II is traditionally used by technicians for reading fault codes and monitoring the actual value of each connected control units. There are few other solutions already available, but none of them satisfies requirements for experienced users. Ordinarily, these available solutions can be applied for monitoring the state of charge and battery parameters. The extraction of all the available data in EV CAN network has been done systematically through OBD-II port and by using low-level software libraries and programming a development board based on ARM STM32F103RBT6 micro-controller. This paper discuss an implementation an integrated OBD-II system for monitoring CAN data such as SOC and Energy consumption based on Android. Finally, the CAN data (e.g., lock and unlock door and Air condition control) will be injected in EV CANs system. Overall, the proposed approach can facilitate the monitoring and control processes for most of specific EV parts considering flexibility and ease of use for the end users.
\end{abstract}

Keyword: Electric vehicle CAN; CAN protocol; EV OBD-II; ARM CortexM-3; Inject CAN data 